\title{
A new piece of an old puzzle: lack of association between C-Rel (rs13031237- rs842647) single nucleotide polymorphisms and non-segmental vitiligo
}

\author{
Eman Salah ${ }^{1^{*}}$ and Alshymaa A. Ahmed ${ }^{2}$
}

\begin{abstract}
Background: The exact pathogenesis of vitiligo is still unclear; however, studies mostly support autoimmune mechanisms including altered T-regulatory cells and FOXP3. C-Rel is a NF-KB family member affecting the normal development of FOXP3 ${ }^{+}$regulatory T cells.

Methods: The aim was to examine the association between 2 C-Rel gene polymorphisms (rs13031237 and rs842647) and non-segmental vitiligo patients. Genomic DNA was isolated from blood samples of 100 patients plus 100 controls for genotyping using restriction fragment length polymorphism and DNA sequencing analyses. Statistical analysis was performed using SPSS program version 21 (IBM Corp., Chicago, IL, USA).

Results: The genotype frequencies did not differ significantly from non-segmental vitiligo patients to controls for both alleles.

Conclusions: C-Rel (rs13031237 and rs842647) polymorphisms are not associated with increased risk for nonsegmental vitiligo. We recommend testing additional mutations in vitiligo patients from different populations to unravel Rel aspects among different autoimmune disorders.
\end{abstract}

Keywords: Melanocytes, Depigmentation, Autoimmune, T-regulatory cells, FOXP3

\section{Background}

Rel is a transcription factor encoded by C-Rel gene that is located on chromosome 2p16.1. It belongs to nuclear factor kappa-light-chain-enhancer of activated B cell $(\mathrm{NF}-\mathrm{KB})$ family, and its signaling pathway plays a critical role in several autoimmune disorders (Chen et al. 2016). C-Rel is expressed utmost only in mature hemopoietic cells but is not necessary for normal hematopoiesis and lymphopoiesis according to C-Rel knockout mice studies. However, C-Rel stays a prerequisite for various specific functions in mature $\mathrm{T}$ and $\mathrm{B}$ cells (Gilmore and Gerondakis 2011) such as antigen presentation and

\footnotetext{
* Correspondence: esmohamed@zu.edu.eg; connexin1980@yahoo.com 1 Dermatology, Venereology \& Andrology department, Faculty of Medicine, Zagazig University, Zagazig, Egypt

Full list of author information is available at the end of the article
}

CD40 signaling cascade (Chen et al. 2016). Furthermore, C-Rel is present at smaller levels in normal endothelial and epithelial cells including the skin. In the skin, loss of NF- $\mathrm{kB}$ activity is known to initiate a strong inflammatory response (Gilmore and Gerondakis 2011). Subsequently, C-Rel has been linked as a susceptibility locus to some autoimmune diseases including systemic lupus erythematosus (Zhou et al. 2012), rheumatoid arthritis (Gregersen et al. 2009; Eyre et al. 2010; Ali et al. 2013), Behcet's disease (Chen et al. 2016), psoriasis (Ali et al. 2013), and celiac disease (Gilmore and Gerondakis 2011; Varadé et al. 2011).

Vitiligo is a de-pigmenting skin disorder resulting in selective destruction of melanocytes. The exact pathogenesis of vitiligo is not clear; however, autoimmunity has been strongly implicated (Dwivedi et al. 2015). The first observation suggesting the involvement of defective T-regulatory cells (Tregs) in vitiligo originated after 
experiments on murine melanomas. In those studies, reduction of Tregs count triggered the generation of cytotoxic $\mathrm{T}$ cells against melanoma cells. This ended in the successful destruction of melanoma, however unexpectedly triggered vitiliginous lesions (Zhang et al. 2007). Additionally, histopathological examination of lesional skin in vitiligo has shown increased CD8+ cytotoxic $\mathrm{T}$ lymphocytes and decreased CD4+ CD25+ FOXP3+ Tregs. This may explain the lost tolerance toward melanocytes with anti-melanocyte reactivity in non-segmental vitiligo (Jahan et al. 2013).

Among different markers expressed by Tregs, FOXP3 is by far the most distinctive (de Boer et al. 2007). As a transcription factor, FOXP3 has a critical role in Tregs differentiation and function. Surprisingly, Ruan et al. 2009 have found that the development of FOXP3+ Tregs requires C-Rel through a complex called the "C-Rel enhanceosome." Additionally, an association between increased susceptibility to non-segmental vitiligo and FOXP3 promoter (rs3761548) polymorphism was found in an Indian population (Jahan et al. 2013).

Herein, we sought to investigate two putative C-Rel single nucleotide polymorphisms (SNPs) (rs13031237 and rs842647) in peripheral blood of non-segmental vitiligo patients as a possible susceptibility risk candidate in comparison to healthy controls. The choice of these SNPs is based on the consideration of previously reported genetic associations with other autoimmune diseases (Chen et al. 2016; Gilmore and Gerondakis 2011; Zhou et al. 2012; Gregersen et al. 2009; Eyre et al. 2010; Varadé et al. 2011; Ali et al. 2013), and rs13031237 and rs 842647 are the most frequently reported SNPs. The SNP rs13031237 is a $\mathrm{G} \rightarrow \mathrm{T}$ transition located in the fourth intron of $R E L$ gene (chromosomic location 61136129). The SNP rs842647 is an A $\rightarrow$ G transition located in the second intron of $R E L$ gene on chromosome 2 (chromosomic location 61119471).

\section{Methods}

\section{Ethical approvals}

The Institutional Review Board (IRB) and ethical committee at Zagazig University Hospitals approved this work. Before enrollment, all subjects provided a wellinformed and written consent.

\section{Subjects}

This study includes a 100 of clinically diagnosed nonsegmental vitiligo patients recruited from the outpatient clinic at the Dermatology, Venereology and Andrology Department of our Hospital. All patients underwent complete history taking including age, sex, disease onset, family history of vitiligo, associated disorders, disease severity using vitiligo area scoring index (VASI), and stability or activity. Stable vitiligo was considered in lack of new lesions, a progression of existing lesions, depigmentation after successful re-pigmentation and of Koebner phenomenon during the last 6 months. All included patients were not receiving any therapy for vitiligo for at least 6 months before analysis. The control group involved one 100 healthy age and sex-matched volunteers. Initially, we tested 30 patients and 30 controls for C-Rel mutations (unpublished work) by PCR, then we extended the subjects count and confirmed the first provisional findings by DNA sequencing as follows.

\section{C-Rel gene polymorphism genotyping by PCR-RFLP}

Two milliliters of anticoagulated whole-blood samples using EDTA were withdrawn from every subject. C-Rel gene single nucleotide polymorphisms (SNPs) (rs13031237 \& rs842647) were genotyped as follows: (1) Genomic DNA extraction was obtained using (QIAamp Blood Kit; Qiagen $\mathrm{GmbH}$, Hilden, Germany) spin column technique, following the manufacturer's guidelines. (2) Amplification of the gene targets by PCR was carried out according to Persico et al. 2006 with modification. The primers for rs13031237 were 5'-GAGTTGTTATGAGAGTAAAAGG CTGC-3' and 5'-AAGTACACAAGTTCTGCCTAGGGT AA-3'. While those for $r s 842647$ were $5^{\prime}$-TGCTTG TCTCTGATTCTCTGGGTC-3' and $5^{\prime}$-CTGGGCGACA AGTGTGAAA CTC-3'. PCR was carried out in $25 \mu \mathrm{l}$ reaction mixture containing $12.5 \mu \mathrm{L}$ of MyTaq, Red Master Mix (bioline, UK), 10 pmol of each primer, $100 \mathrm{ng}$ of genomic DNA, and nuclease-free water up to $25 \mu \mathrm{l}$. PCR began first with denaturation at $95{ }^{\circ} \mathrm{C}$ for $5 \mathrm{~min}$, then 35 cycles of denaturation at $95^{\circ} \mathrm{C}$ for $30 \mathrm{~s}$, annealing at 62 or $63{ }^{\circ} \mathrm{C}$ for rs 13031237 and rs842647, respectively, for $40 \mathrm{~s}$, and $72{ }^{\circ} \mathrm{C}$ for $30 \mathrm{~s}$, followed by the last extension step at $72{ }^{\circ} \mathrm{C}$ for $3 \mathrm{~min}$. PCR yields were checked out on $2.0 \%$ agarose gel electrophoresis as bands at $268 \mathrm{bp}$ and $193 \mathrm{bp}$ for rs13031237 and rs842647 respectively. (3) Restriction of RFLP products using enzyme Csp6I (Thermo Fisher Scientific Inc., Ontario, Canada) for rs13031237 in $37{ }^{\circ} \mathrm{C}$ and $\mathrm{HpyCH} 4$ III for rs842647 (Thermo Fisher Scientific Inc.,Ontario, Canada) in $65^{\circ} \mathrm{C}$ was according to the manufacturer's guidelines. (4) PCR-RFLP product interpretation. Products were visualized on $2.0 \%$ agarose gel stained with ethidium bromide on an ultraviolet transilluminator. The candidate mutations in this study came at the cut point of the used restriction enzymes, so the wild alleles will be digested while the mutated ones will not. Homozygous wild genotypes will appear as two bands which are shorter than the original amplification band. While homozygous mutated genotypes will appear as one band at the site of the amplified one. A heterozygous case will appear as three bands, i.e., for rs13031237, they will be at 79 and 189 bp while for rs842647, they will be at 58 and $135 \mathrm{bp}$. While homozygous mutated genotypes will appear as one band 
at the site of the amplified one. A heterozygous case will appear as three bands.

\section{REL gene polymorphism genotyping by DNA sequencing} Ambiguous samples were genotyped and 10\% of samples were double-checked using direct Sanger sequencing as follows: (1) TopTaq Master Mix Kit (250) (Qiagen $\mathrm{GmbH}$, Hilden, Germany) in $50 \mathrm{uL}$ reaction volume with the previously mentioned protocol was used for PCR amplification. (2) Amplified PCR products underwent the first purification using QIAquik PCR Purification Kit (50) (Qiagen $\mathrm{GmbH}$, Hilden, Germany) using the manufacturer's guidelines. (3) Cycle sequencing performed using Bigdye Terminator V3.1 cycle sequencing kit (Thermo Fisher Scientific Inc., Ontario, Canada) followed by the second purification of the products using BigDye $\mathrm{X}$ Terminator Purification Kit (Thermo Fisher Scientific Inc., Ontario, Canada), according to the manufacturer's guidelines. (4) The Applied Biosystems 3500 Genetic Analyzer (Thermo Fisher Scientific Inc., Ontario, Canada) was used for sequencing of the purified products. (5) Nucleotide Blast online program (https://blast.ncbi.nlm.nih.gov) was used for data analysis and interpretation of results.

\section{Statistical analysis}

SPSS program version 21 (IBM Corp., Chicago, IL, USA) was used for statistical analysis. Hardy-Weinberg equilibrium was conducted on the control group by using the chi-square test. Frequencies of each genotype, as well as allele, were expressed as numeral and percentage. Differences in C-Rel allele frequencies were analyzed using the chi-square test and the two-tailed Fisher's exact test for the results $\leq 5$. The odds ratios (ORs), as well as the 95\% confidence intervals, were calculated. $P \leq 0.05$ was considered as an indicator for statistically significant differences.

\section{Results}

This work included 70 females and 30 males with nonsegmental vitiligo as well as a 100 of healthy volunteers as controls. Patients' ages ranged from 9 to 55 years old with a mean of 29.0 years, and VASI scores ranged from 20 to 70 . Table 1 describes the clinical characteristics of the studied patients. Genotypes and allele distribution of the two tested SNPs among patients and controls were summarized in Table 2 and Fig. 1. There was no significant association between these mutations and disease occurrence. Independent sample $t$ test was used to compare the mean age of onset of positive versus negative patients for each SNP to clarify whether these mutations affect the age of onset of vitiligo or not. For rs13031237, the mean age of onset of positive patients (GT and TT genotypes) was 30 years old while for the negative cases (GG genotype), it was 21.5 years old $(p=0.06)$; for
Table 1 Clinical characteristics of non-segmental vitiligo patients

\begin{tabular}{lll}
\hline Character & $n$ (total = 100) & $\%$ \\
\hline Females & 70 & 70 \\
Males & 30 & 30 \\
Family history for vitiligo & 30 & 30 \\
Associated other disorders & & \\
$\quad$ Diabetes mellitus type-1 & 15 & 15 \\
$\quad$ Hypothyroidism & 5 & 5 \\
Stable vitiligo & 40 & 40 \\
Active vitiligo & 60 & 60 \\
\hline
\end{tabular}

rs842647, the mean ages were 27 and 24.1 years old for AG and GG, and AA, respectively, $(p=0.7)$. Also, there was no significant association between the studied mutations and disease activity. It is worth mentioning that 20 patients have an associated disease (diabetes mellitustype1 or hypothyroidism) and are positive for rs13031237 (GT genotype).

\section{Discussion}

In the present study, we have tested the possible link between C-Rel SNPs and non-segmental vitiligo. We decided to analyze the most commonly reported C-Rel mutations to be associated with other autoimmune disorders. Two specific mutations (namely, rs13031237 and rs842647) were more frequently associated with immune-mediated diseases (Chen et al. 2016) such as celiac disease (Trynka et al. 2009), rheumatoid arthritis (Gregersen et al. 2009; Eyre et al. 2010; Varadé et al. 2011), SLE (Zhou et al. 2012), psoriasis (Ali et al. 2013), and Behcet's disease (Chen et al. 2016). In spite the fact that "rs13031237" is the most commonly studied C-Rel SNP in autoimmune diseases, it was also interesting to study rs842647 in vitiligo, as its GG genotype and G

Table 2 Frequencies of alleles and genotypes of REL polymorphisms in non-segmental vitiligo patients and controls

\begin{tabular}{llllll}
\hline SNP & $\begin{array}{l}\text { Genotype/ } \\
\text { allele }\end{array}$ & $\begin{array}{l}\text { Cases } \\
n=100\end{array}$ & $\begin{array}{l}\text { Controls } \\
n=100\end{array}$ & $P$ value & $\begin{array}{l}\text { OR } \\
(95 \% \mathrm{Cl})\end{array}$ \\
\hline rs13031237 & GG & 53 & 46 & 0.3 & $1.3(0.75-2.3)$ \\
& GT & 40 & 46 & 0.4 & $0.8(0.44-1.4)$ \\
& TT & 7 & 8 & 0.8 & $0.9(0.3-2.4)$ \\
& G & 93 & 92 & 0.5 & $1.1(0.7-1.9)$ \\
& rs842647 & 47 & 54 & 0.5 & $0.8(0.5-1.4)$ \\
& AA & 75 & 69 & 0.3 & $1.3(0.7-2.5)$ \\
& AG & 20 & 30 & 0.1 & $0.6(0.3-1.1)$ \\
& GG & 5 & 1 & 0.1 & $5.2(0.6-45)$ \\
& A & 95 & 99 & 0.6 & $1.1(0.7-2.2)$ \\
& G & 25 & 31 & 0.6 & $0.8(0.5-1.5)$ \\
\hline
\end{tabular}

SNP single nucleotide polymorphism, $O R$ odds ratio, $\mathrm{Cl}$ confidence interval 


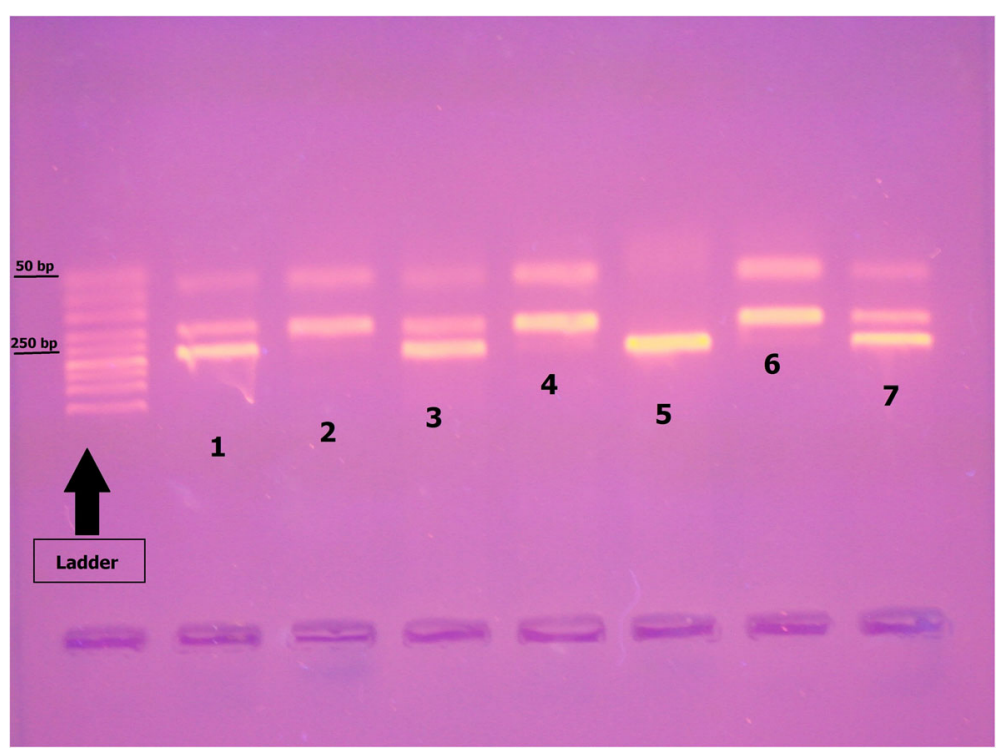

Fig. 1 Agarose gel electrophoresis for restriction fragments of C-Rel gene (rs 13031237). (Chen et al. 2016; Zhou et al. 2012; Ali et al. 2013) heterozygous (GT genotype), (Gilmore and Gerondakis 2011; Gregersen et al. 2009; Varadé et al. 2011) homozygous wild (GG genotype), (Eyre et al. 2010) homozygous mutated (TT genotype)

allele was associated with a more risk for cutaneous lesions in patients with Behcet's disease. This finding may indicate that rs 842647 polymorphism can affect the occurrence of autoimmune disease as well as the tendency to show skin manifestations (Chen et al. 2016).

In the present work, we could not detect a significant association between the two tested C-Rel SNPs and nonsegmental vitiligo in the study population. Perhaps other different and less common Rel mutations are linked to non-segmental vitiligo which necessitates further studies. Another possible explanation would be a different and distinct unknown autoimmune cascade that is involved in vitiligo and not in other autoimmune disorders. Also, previous conflicting results about the role of Rel in mouse and human models have been reported for both diabetes mellitus and bronchial asthma (Fullard et al. 2012). Herein, it is worth mentioning that 20 of our patients who have an associated diabetes mellitus-type1 or hypothyroidism are positive for rs13031237 (GT genotype) which have been linked previously to SLE (Zhou et al. 2012), rheumatoid arthritis (Gregersen et al. 2009; Eyre et al. 2010; Varadé et al. 2011), psoriasis (Ali et al. 2013), and celiac disease (Trynka et al. 2009). We believe that it would be more interesting if we can test such cases for presence or absence of autoantibodies, e.g., anti-islet cell antibodies and anti-thyroid antibodies. Furthermore, we could not find previous studies about Rel and hypothyroidism which would be a good research point for interested colleagues.

Vitiligo is an old puzzle and different hypotheses have been suggested to explain its pathogenesis (Iannella et al.
2016). Recently, the role of Tregs has been linked to vitiligo pathogenesis. Several studies revealed either reduction of Tregs count in peripheral blood and/or functional abnormalities in vitiligo patients. These deviations may increase the count and activity of cytotoxic $\mathrm{T}$ cells leading to loss of melanocytes (Dwivedi et al. 2015). The "enhanceosome" model described by Ruan et al. 2009 perfectly explains the link between C-Rel and the development of FOXP3+ Tregs. They proposed that antigen-presenting cells can bind to TCR and CD28 in the presence of TGF$\beta$. This binding leads to the activation of $I K K b$, which phosphorylates $\mathrm{IkB} \alpha$ resulting in C-Rel and p65-free release. Then "C-Rel-p65" dimer migrates to the nucleus and attaches to a FOXP3 promoter. Finally, this prompts the creation of Tregs-specific multifactorial transcriptional complex or the "enhanceosome" to switch on/or off Tregs differentiation (Ruan et al. 2009). Furthermore, Grigoriadis et al. 2011 reports the requirement of C-Rel for conversion of FOXP3- Tregs precursors into FOXP3+ Tregs through IL-2 and IL-15. In vitiligo, cumulative observations indicated an impaired FOXP3 expression that might disturb Tregs norms (Dwivedi et al. 2015).

However, the lack of association between the two tested C-Rel SNPs in our study population could also be a similar finding to some previous studies who failed to replicate the association of C-Rel with SLE (Zhou et al. 2012; Varadé et al. 2011; Cen et al. 2013) in contrast to several other studies. To add more complexity, C-Rel may show diverse patterns among different autoimmune disorders and even within the same immune-mediated disorder in different populations. 


\section{Conclusions}

To our knowledge, this is the first study to test for the potential link between C-Rel SNP and non-segmental vitiligo. Lack of previous reports was a limitation to compare our data to other studies. So, we highly recommend testing further C-Rel mutations in non-segmental vitiligo and in different populations. Finally, this would provide an explanation for the controverting behavior of C-Rel among different autoimmune disorders.

\section{Abbreviations}

DNA: Deoxyribonucleic acid; EDTA: Ethylenediaminetetraacetic Acid; FOXP3: Forkhead box P3; IkBa: Nuclear factor of kappa light polypeptide gene enhancer in B-cells inhibitor alpha; IKKb: Inhibitor of nuclear factor kappa-B kinase; IL: Interleukin; IRB: Institutional review board; NF-kB: Nuclear factor kappa-light-chain-enhancer of activated B cells; PCR-RFLP: Polymerase chain reaction-restriction fragment length polymorphism; SLE: Systemic lupus erythematosus; SNP: Single nucleotide polymorphism; Tregs: T-regulatory cells; VASI: Vitiligo area scoring index

\section{Acknowledgements}

All molecular techniques performed at Zagazig University Hospitals, Clinical Pathology Department, Immunology, and Research Unit Laboratory.

\section{Funding}

This research did not receive any specific grant from funding agencies in the public, commercial, or not-for-profit sectors.

\section{Availability of data and materials}

Not applicable.

\section{Authors' contributions}

Both authors read and approved the final manuscript. ES was responsible for the research idea, the patients' enrollment, and writing the major part of the manuscript. AA was responsible for the Lab section for the gene study and statistical analysis and shared in writing as well as revising the manuscript.

\section{Ethics approval and consent to participate}

The Institutional Review Board (IRB) and ethical committee at Zagazig University Hospitals approved this work. Before enrollment, all subjects provided a well-informed and written consent.

\section{Consent for publication}

Not applicable.

\section{Competing interests}

The authors declare that they have no competing interests.

\section{Publisher's Note}

Springer Nature remains neutral with regard to jurisdictional claims in published maps and institutional affiliations.

\section{Author details}

'Dermatology, Venereology \& Andrology department, Faculty of Medicine, Zagazig University, Zagazig, Egypt. ${ }^{2}$ Clinical Pathology department, Faculty of Medicine, Zagazig University, Zagazig, Egypt.

Received: 15 December 2017 Accepted: 23 March 2018

Published online: 15 June 2018

\section{References}

Ali FR, Barton A, Smith RL, et al. An investigation of rheumatoid arthritis loci in patients with early onset psoriasis validates association of the REL gene. $\mathrm{Br}$ J Dermatol. 2013;168(4):864-6.

Cen $\mathrm{H}$, Zhou M, Leng RX, et al. Genetic interaction between genes involved in NF-KB signaling pathway in systemic lupus erythematosus. Mol Immunol. 2013;56(4):643-8
Chen F, Xu L, Zhao T, et al. Genetic variation in the REL gene increases risk of Behcet's disease in a Chinese Han population but that of PRKCQ does not. PLoS One. 2016;11(1):e0147350.

de Boer OJ, van der Loos CM, Teeling P, et al. Immunohistochemical analysis of regulatory T cell markers FOXP3 and GITR on CD41CD251 T cells in normal skin and inflammatory dermatoses. J Histochem Cytochem. 2007:55:891-8.

Dwivedi M, Kemp EH, Laddha NC, et al. Regulatory T cells in vitiligo: implications for pathogenesis and therapeutics. Autoimmun Rev. 2015;14:49-56.

Eyre S, Hinks A, Flynn E et al 2010. Confirmation of association of the REL locus with rheumatoid arthritis susceptibility in the UK population. Ann Rheum Dis 2010;69(8):1572-1573

Fullard N, Wilson CL, Oakley F. Roles of c Rel signalling in inflammation and disease. Int J Biochem Cell Biol. 2012;44(6):851-60.

Gilmore TD, Gerondakis S. The c-Rel transcription factor in development and disease genes cancer. 2011;2(7):695-711.

Gregersen PK, Amos Cl, Lee AT, et al. 2009. REL, encoding a member of the NFkappaB family of transcription factors, is a newly defined risk locus for rheumatoid arthritis. Nat Genet. 2009;41(7):820-3.

Grigoriadis G, Vasanthakumar A, Banerjee A, et al. c-Rel controls multiple discrete steps in the thymic development of Foxp3 (+) CD4 regulatory T cells. PLoS One. 2011;6(10):e26851.

lannella G, Greco A, Didona D, et al. Vitiligo: pathogenesis, clinical variants and treatment approaches. Autoimmun Rev. 2016;15(4):335-43.

Jahan P, Cheruvu R, Tippisetty S, et al. Association of FOXP3 (rs3761548) promoter polymorphism with nondermatomal vitiligo: a study from India. J Am Acad Dermatol. 2013;69(2):262-6.

Persico M, Capasso M, Persico E, et al. Interleukin-10-1082 GG polymorphism influences the occurrence and the clinical characteristics of hepatitis $C$ virus infection. J Hepatol. 2006;45(6):779-85.

Ruan Q, Kameswaran V, Tone Y, et al. Development of Foxp3 (+) regulatory t cells is driven by the c-Rel enhanceosome. Immunity. 2009;31(6):932-40.

Trynka G, Zhernakova A, Romanos J, et al. Coeliac disease associated risk variants in TNFAIP3 and REL implicate altered NF kappaB signaling. Gut. 2009;58(8): 1078-83.

Varadé J, Palomino-Morales R, Ortego-Centeno N, et al. Analysis of the REL polymorphism rs13031237 in autoimmune diseases. Ann Rheum Dis. 2011; 70(4):711-2.

Zhang P, Côté AL, de Vries VC, et al. Induction of postsurgical tumor immunity and T-cell memory by a poorly immunogenic tumor. Cancer Res. 2007;1:6468-76.

Zhou XJ, Lu XL, Nath SK, et al. Gene-gene interaction of BLK, TNFSF4, TRAF1, TNFAIP3, and REL in systemic lupus erythematosus. Arthritis Rheum. 2012; 64(1):222-31.
Submit your next manuscript to BioMed Central and we will help you at every step:

- We accept pre-submission inquiries

- Our selector tool helps you to find the most relevant journal

- We provide round the clock customer support

- Convenient online submission

- Thorough peer review

- Inclusion in PubMed and all major indexing services

- Maximum visibility for your research

Submit your manuscript at www.biomedcentral.com/submit
Biomed Central 